\title{
Computational Study for Workforce Scheduling and Routing Problems
}

\author{
J. Arturo Castillo-Salazar, Dario Landa-Silva and Rong Qu \\ Automated Scheduling, Optimisation and Planning (ASAP) Research Group \\ School of Computer Science, Jubilee Campus, The University of Nottingham, Wollaton Road, NG8 1BB, Nottingham, UK \\ (psxjaca, dario.landasilva, rong.qu)@nottingham.ac.uk
}

Keywords: Workforce Scheduling and Routing, Mathematical Programming, Benchmark Datasets and Results

Abstract: We present a computational study on 112 instances of the Workforce Scheduling and Routing Problem (WSRP). This problem has applications in many service provider industries where employees visit customers to perform activities. Given their similarity, we adapt a mathematical programming model from the literature on vehicle routing problem with time windows (VRPTW) to conduct this computational study on the WSRP. We generate a set of WSRP instances from a well-known VRPTW data set. This work has three objectives. First, to investigate feasibility and optimality on a range of medium size WSRP instances with different distribution of visiting locations and including teaming and connected activities constraints. Second, to compare the generated WSRP instances to their counterpart VRPTW instances with respect to their difficulty. Third, to determine the computation time required by a mathematical programming solver to find feasible solutions for the generated WSRP instances. It is observed that although the solver can achieve feasible solutions for some instances, the current solver capabilities are still limited. Another observation is the WSRP instances present an increased degree of difficulty because of the additional constraints. The key contribution of this paper is to present some test instances and corresponding benchmark study for the WSRP.

\section{INTRODUCTION}

A lot of research has been dedicated to the Vehicle Routing Problem with Time Windows (VRPTW). Research literature on workforce scheduling is also abundant. For these problems, benchmark instances and results have been published, which can be used to assess the performance of new proposed solution methods. This is not the case for the combined problem of Workforce Scheduling and Routing. To the best of our knowledge, there are no benchmark datasets that have been studied to the same extent as VRPTW and workforce scheduling independently. Our long term goal is to develop powerful algorithms to tackle WSRP. This study represents our first step towards achieving that goal. Here, we take some well-known VRPTW instances (Solomon, 1987) and include additional data to transform them into WSRP instances. We then conduct computational experiments to assess the difficulty of these generated WSRP instances compared against the original VRPTW ones. We use a modern mathematical programming solver in our experiments allowing us to obtain an updated perspective of the capability of such solution method to tackle the WSRP and establishing a base-line to compare future work.
We use the term Workforce Scheduling and Routing Problem (WSRP) in reference to problems where employees travel across different locations to perform their work. WSRPs combine characteristics of the general employee scheduling problem (Glover and McMillan, 1986) and vehicle routing problem with time windows (VRPTW) (Solomon, 1987) to describe the work of mobile employees in an organisation. Some service provider-industries require employees to visit customers at their premises to perform specific activities. Examples of such service industries include healthcare, security, communications, domestic cleaning, etc. Customers are located at different addresses and require service within a time window or other timing requirement. In most cases, travelling time is considered part of employees' working time. As a result, reductions in travel time could mean more time to perform additional customers visits. Scheduling employees to meet these time windows on visits whilst reducing travel time is a difficult process for medium to large size scenarios. WSRP's size is often given by the number of employees and the number of activities.

Although at first, WSRP could be seen as identical to VRPTW, there are important differences. In VRPTW scenarios, the number of vehicles is usually 
not fixed (minimising the number of vehicles is often the goal) and vehicles types are often the same. Nevertheless, research on scenarios with heterogeneous fleet of vehicles (Dondo and Cerdá, 2007) and where the number of vehicles is limited (Chuin Lau et al., 2003) has also been reported. In WSRP scenarios, the number of employees is usually known in advance. An objective is often to balance the workload instead of reducing the number of workers. Workforce is heterogeneous since every employee is different considering their individual skills, training, attitudes, etc.

Castillo-Salazar et al. (2012) describe WSRP's main characteristics. Time window refers to the period by which the service should start. Start and end location indicate if employees start/end their work at home, central building, etc. Service time refers to activities' duration at customer premises. Skills reflect the potential diversity of abilities in the workforce. Transportation mode specifies if it is possible to use more than one medium of transportation between the visiting locations, i.e. walking, car, public transport, etc. Connected activities handle all time dependant relations between two or more activities, for example synchronisation and precedence. Teaming considers activities needing a team of employees. Clustering, refers to forming groups of visits based on geographical location usually to reduce the size of the problem.

Given the similarity between WSRP and VRPTW, researchers have successfully utilized VRPTW models and solution techniques to obtain feasible solutions for WSRP-like scenarios. For example, home healthcare (Cheng and Rich, 1998; An et al., 2012; Nickel et al., 2012), patrolling of security officers (Misir et al., 2011; Chuin Lau and Gunawan, 2012), engineers/technicians on field (Günther and Nissen, 2012). These previous works cover: time windows, start/end location, skills, service time and transportation mode. Other characteristics such as connected activities, teaming and clustering have been researched to a lesser extent in the WSRP literature. There are some exceptions, for example, connected activities have been considered by Rasmussen et al. (2012), while teaming has been considered in Li et al. (2005) and Dohn et al. (2009).

Connected activities and Teaming are important features of WSRP because they allow the modelling of scenarios with linked activities. Depending on the service sector, this could be for example, conducting a 2nd visit within a day to administer an additional medication dose to the patient, or bringing two specialist technicians at the same time to install and calibrate equipment, etc. These constraints already exist in the VRPTW literature (Toth and Vigo, 1987; Taillard et al., 1996; Bredström and Rönnqvist, 2008).
Bredström and Rönnqvist (2008) apply their mathematical model of VRPTW with temporal precedence and synchronisation constraints to tackle home healthcare and forest operations, which are examples of WSRP scenarios. In their experiments, the majority of instances have 20 visits only and all visits are uniformly distributed in a square area, hence with no apparent clusters of visits. As part of our study we adapt 112 VRPTW instances, 56 of them contain 25 visits and the remaining 56 contain 50 visits. Additionally, clusters of visits are present in half of the instances. This brings us closer to having problem instances reflecting real-world WSRP scenarios.

Our study has three objectives. The first objective is to use Bredström and Rönnqvist (2008) VRPTW model to tackle medium size WSRP instances (including 20 or more visits). The second objective is to assess the difficulty of the adapted problem instances as a result of adding connected activities and teaming constraints. We aim to test by experimentation whether WSRP is a more difficult problem to solve than traditional VRPTW for a mathematical programming solver. The third objective is to establish the computational time that a mathematical solver needs to find feasible solutions, if such solutions exist, for our adapted data set. Regarding the third objective, most research papers report computation time within minutes when solving small instances. Using a solver to obtain optimal solutions in medium to large instances has been reported to take up to 64 hours ( $\mathrm{Li}$ et al., 2005). Commonly, real-world WSRP scenarios have a planning time horizon of one day, and the problem can be solved at the beginning of the working day or at the end of the previous one. In such scenarios, waiting many hours to obtain a solution is not practical. Therefore, our experiments consider three different computation time settings for the mathematical solver: $15 \mathrm{~min}, 60 \mathrm{~min}$ and $240 \mathrm{~min}$.

The remaining of the paper has 4 sections. Section 2 explains Bredström and Rönnqvist (2008) model, and the adaptations performed for tackling WSRP. Section 3 describes the data set used and how it is generated from the original widely known Solomon (1987) instances. Section 4 describes our experiments and results, divided in 3 sub-sections, each of them focusing in one of the three objectives stated above. Finally, section 5 provides our conclusions.

\section{INTEGER LINEAR MODEL}

The integer linear programming model used for the present computational study is the one by Bredström and Rönnqvist (2008). The model was chosen because it considers synchronisation and precedence constraints. Both constraints are necessary to model 
situations in which more than one employee need to arrive at a location at the same time or when activities depend on the finishing/starting time of other activities. There are other models in the literature that contain these constraints, for example the one by Korsah et al. (2010). But the Korsah et al. model uses more variables to capture the waiting time of vehicles. Another model including synchronisation and precedence constraints is the one by Rasmussen et al. (2012), which also uses more variables to record if an activity is performed or not by an employee. We aim to schedule all activities, otherwise we consider the scenario as infeasible. Such infeasible scenarios occur due to limited number of workers, and the availability or lack of skills. The extra variables in the models by Korsah et al. and Rasmussen et al. require much more memory when tackled by the solver. Since the features modelled with those extra variables are not required in our study we opt for the Bredström and Rönnqvist model.

In the WSRP the number of employees is limited. The set $N$ is all clients locations. Then, $o, d$ represent the same location (start and end) but two different nodes are required for modelling purposes. $A$ is a set containing all clients locations $N$ plus $o, d . K$ is the set of all employees. Time windows are given with the values $a_{i}$ for the earliest start time and $b_{i}$ for the latest start time of visit $i$. All visits have a set duration given by $D_{i}$. Travel time between visits $i$ and $j$ is a predefined value $T_{i j}$. Variables $t_{i k}$ contain the time when visit $i$ starts, performed by employee $k$, all times are given in minutes after the start of the time horizon. Finally, each employee has a time window representing their working time. Terms $a_{i}^{k}$ and $b_{i}^{k}$ represent the start and the end of the working time for employee $k$ respectively. The term $E_{i j}$ includes the travelling time from visit $i$ to visit $j$ plus the duration of visit at node $i\left(E_{i j}=T_{i j}+D_{i}\right)$.

The objective function (1) has two elements: the cost for assigning a preferred visit to an employee $c_{i k}$ and the travel time of all employees when performing their visits. In the original model by Bredström and Rönnqvist there is a third element to balance distance and time. Such element is not used here because distance and travel time between visits or locations are equal in the Solomon instances. We set the weights in the objective function (1) to the same value $\alpha_{p}=\alpha_{T}$. All visits need to be performed (2). All employees must start and return to the initial location after the last visit $o, d$ (3). Constraint (4) preserves employees' flow conservation. Every visit time window must be satisfied (5, 6 and 7). All visits need to be performed during the employees starting and ending times (8). When two or more visits require starting at the same time, a synchronisation constraint (9) is necessary for every pair of visits. Other type of temporal dependencies are covered by constraint (10). The binary decision variables $x_{i j k}$ are set to 1 if employee $k$ travels from $i$ to $j$ and are set to 0 otherwise (11). Apart from the objective function, another change made to the model is in the scheduling variables $t_{i k}$. In the original model the scheduling variables are positive real numbers. Here, the scheduling variables are enforced to be positive integers (12). Such change makes the problem harder to solve but provides an exact time for the activities without introducing rounding errors for seconds and milliseconds. In our data sets, all employees have the same starting and ending time which matches the time horizon of every instance.

$$
\begin{gathered}
\min \quad \alpha_{p} \sum_{k \in K} \sum_{(i, j) \in A} c_{i k} x_{i j k}+\alpha_{T} \sum_{k \in K} \sum_{(i, j) \in A} T_{i j} x_{i j k} \\
\text { s.t. } \sum_{k \in K} \sum_{j:(i, j) \in A} x_{i j k}=1 \quad \forall i \in N, \\
\sum_{j:(o, j) \in A} x_{o j k}=\sum_{j:(j, d) \in A} x_{j d k}=1 \quad \forall k \in K, \\
\sum_{j:(i, j) \in A} x_{i j k}-\sum_{j:(j, i) \in A} x_{j i k}=0 \forall i \in N, \quad \forall k \in K, \\
t_{i k}+E_{i j} x_{i j k} \leq t_{j k}+b_{i}-b_{i} x_{i j k} \forall k \in K, \forall i, j \in A, \\
a_{i} \sum_{j:(i, j) \in A} x_{i j k} \leq t_{i k} \quad \forall k \in K, \quad \forall i \in N, \\
t_{i k} \leq b_{i} \sum_{j:(i, j) \in A} x_{i j k} \quad \forall k \in K, \quad \forall i \in N, \\
a_{i}^{k} \leq t_{i k} \leq b_{i}^{k} \quad \forall k \in K, \quad \forall i \in o, d, \\
\sum_{k \in K} t_{i k}=\sum_{k \in K} t_{j k} \quad \forall i, j \in P^{s y n c}, \\
\sum_{k \in K} t_{i k}+p_{i j} \leq \sum_{k \in K} t_{j k} \forall i, j \in P^{p r e c}, \\
x_{i j k} \in\{0,1\} \forall k \in K, \forall i, j \in A, \quad \forall \\
t_{i k} \in \mathbb{Z}_{+} \forall k \in K, \quad \forall i \in N .
\end{gathered}
$$

\section{WSRP PROBLEM INSTANCES}

\subsection{Description of Solomon's Instances}

Solomon (1987) data set for the VRPTW has been used broadly in the literature. There are 56 instances grouped according to two criteria: varying the planning horizon and visits-location. Combining these criteria forms 6 groups: R100, R200, C100, C200, RC100, RC200. Groups R100 and RC100 have short planning horizon (230 and 240 minutes) and groups RC200, R200, C100 and C200 have longer planning horizons of more than 900 minutes. Considering the location of visits which vehicles have to complete, there are three different types: clusters $(\mathrm{C})$, random 


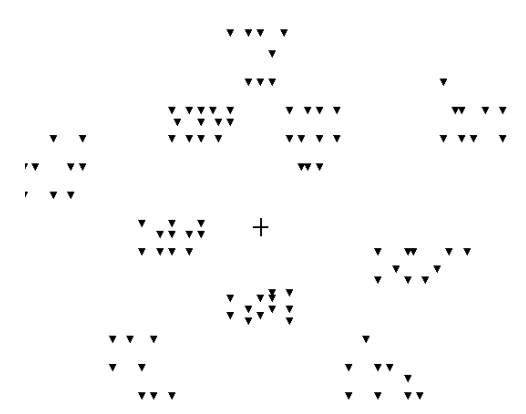

(a) Clustered - C

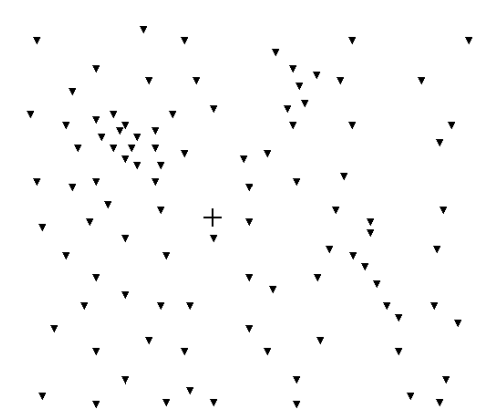

(b) Random - R

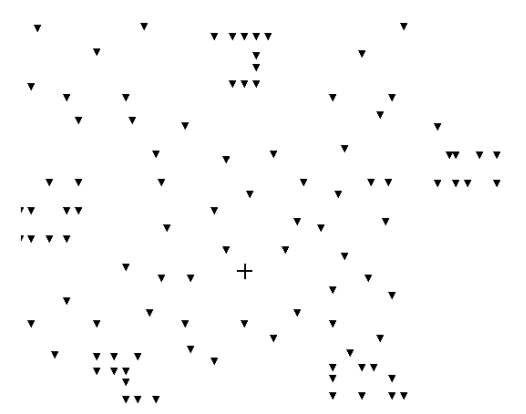

(c) Mixed - RC

Figure 1: Three different types of location arrangement of visits in the Solomon's data set (Solomon, 1987). Original instances with 100 visits each. The depot is identified with a black cross.

(R) and mixed (RC). Figure 1 shows the layout of the instances according to their location of visits. In sub-figure 1a (C group) ten clusters can be appreciated. A random layout of visit locations is present in sub-figure $1 \mathrm{~b}$ ( $\mathrm{R}$ group). Finally, a mixed scenario is shown in sub-figure 1c with five clusters and some random visits (RC group). All instances in each type of visit-location group ( $\mathrm{C}, \mathrm{R}$ and $\mathrm{RC})$ have the same distribution of visits. In total, there are 17 clustered, 23 random and 16 mixed instances. All clustered instances (C) have a visit duration of 90 min while random and mixed instances ( $\mathrm{R}$ and $\mathrm{RC}$ ) have a visit duration of $10 \mathrm{~min}$.

\subsection{Generating WSRP Instances}

We took a data set with 56 instances having 100 visits each. For this study we use versions of each instance with 25 and 50 visits since preliminary experiments with all 100 visits resulted in the solver running out of memory. We took the first 25 and 50 visits in the order in which they appear in the original 100 visit file since as suggested by Solomon, doing this provides a good distribution of visits. In total, 112 instances were generated, 56 instances with 25 visits and 56 instances with 50 visits. Moreover, the 112 instances were adapted to include enough information to be representative of WSRP scenarios. First, in the VRPTW there is no limit in the number of vehicles to complete all visits. In contrast, in WSRP the number of employees is known in advance, perhaps with the exception of casual employees who are called "on demand" if needed. Even though, casual employees can be added for a day or two when necessary, it is often preferred no to use them, and try to complete all visits with the staff available. As a result, we defined a set of workers for every instance by setting the ratio of employees to visits to $1 / 5$. This value was decided following our conversations with a home care provider, and it also matches the assumption by Bredström and Rönnqvist (2008). The home care provider told us that their average visit duration is currently $50 \mathrm{~min}$. At present, the provider gives a maximum of $30 \mathrm{~min}$ for the carer to move from one location to another and carers must start and end their working day at the provider's main site. Current shift length is 8 hours with a break of 1 hour, or two breaks of 30 min each. Taking this into account, we determine the mean visits per employee per day $x$ by solving the simple equation $50 x+30(x+1)+60=480$, where $x+1$ is the number of trips in a route including the last trip returning to the provider's main site. It is assumed that the breaks are taken near a visit. The result is $x=4.875$ rounded to 5 . In other words, instances with 25 activities have a workforce of 5 employees and instances with 50 visits have a workforce of 10 employees. In some instances we further reduced the workforce by one employee emulating an absent employee. The capacity of each vehicle in VRPTW can refer to the number of hours every employee is allowed to work under his/her contractual agreement. In this study, we it is assumed that employees are available to work throughout the time horizon.

Teaming is included in the adapted instances by making a percentage of visits to require more than one employee to be performed. As suggested by Bredström and Rönnqvist (2008, p. 11), we set $10 \%$ of the visits in each instance to requiring two employees for the whole duration of the visit. Employees' preferences are not included in the original Solomon data set but required to compute the objective function (1). For this, we randomly assigned a preference (high, medium, low and not-preferred) value for each employee in regards to each visit.

Finally, the last addition to the data set is connected activities. There are 5 types of connected activities, as defined by Rasmussen et al. (2012): synchronisation, overlapping, minimum difference, maximum difference and min-max difference. Each type relates two visits. Synchronisation when both activities start at the same time. Overlapping, for a period 
of time both activities are being performed simultaneosly. Minimum difference restricts the start of the second activity after a minimum time has passed from the staring of the first one. Maximum time difference limits the start of the second activity from immediately up to a defined value. Finally, min-max difference is a combination of the previous two, with a specific time window (minimum and maximum starting time) for the second activity in relation to the commencing of the first one. The procedure to add connected activities constraints is as follows. Every visit has a $25 \%$ probability of having a connected activity constraint with the activity that immediately follows in the original Solomon's instance. Rasmussen et al. (2012) used three different percentages $10 \%, 20 \%$ and $30 \%$. The visits order remain the same. If there is a connected activity constraint, then we must choose between one of the 5 different types. In real scenarios the two first types (synchronisation and overlapping) occur more often than the last three. Hence, we assigned the following probabilities to each type: $35 \%$ to each synchronisation and overlapping; $10 \%$ to each of maximum, minimum and min-max difference. When using the previously described procedure to create connected activities constraints, sometimes the added constraints make the instance infeasible, e.g. an overlapping constraint for two activities that given their duration and time windows can definitely not overlap. In such cases the constraint is discarded and another one tried.

Changing the Solomon dataset with the adaptations described above, could make the problem instances to become easier to solve than the original ones. The experiments in subsection 4.3 aim to test this possibility by solving the WSRP model with and without the teaming and connected activities constraints. In the following, we refer to the 112 generated problem instances as WSRP instances.

\subsection{Other Instances in the Literature}

There are other problem instances used in published papers relating to problems that can be seen as WSRP. Examples include the Akjiratikarl et al. (2007) data set which is based on a council in Wales scheduling care workers across the region The home health care data set by Bertels and Fahle (2006) is a generated one and it consists of 120 test scenarios containing between 20 and 50 nurses and up to 200 jobs. Such data set does not include any jobs requiring more than one worker (nurse) and there is no presence of connected activities. The manpower allocation problem (Li et al., 2005) contains job-teaming constraints but the number of instances (25) is limited for our study and the number of jobs in each instance is on average
300, which the solver cannot handle. Moreover, there is no set number of workers available since the objective is to find a minimum workforce size. There is also the data set by Cheng and Rich (1998) which is considered too small, with 4 nurses and 10 jobs. Finally, the most complete data set which includes both teaming and connected activities constraints is the one by Rasmussen et al. (2012). In that data set, the majority of instances are understaffed which leaves some activities not performed. The IP Model used here does not consider such possibiliy so it would result in having infeasible instances.

\section{EXPERIMENTS AND RESULTS}

\subsection{Description of Experiments}

Three sets of experimental results are described in the following subsections. The first set relates to the objective of tackling WSRP with the IP model by reporting on gap achieved. The second set addresses the second objective of studying whether or not the additional connected activities and teaming constraints makes the WSRP easier or more difficult to solve. Finally, the third set is associated to the first set to analyse how much time is necessary to find a feasible solution for the adapted instances. Additionally, the third set of experiments also provides an insight into the type of method used by the solver when finding feasible solutions, either through branching-cutting or with heuristics. All the experiments are performed using Gurobi version 5.5 and CPLEX 12.5. The results produced by the two solvers do not differ much. Nevertheless, using our computational setting, Gurobi finds better results for more instances. Therefore, we report the Gurobi results only. No parameter tuning was performed on either of the solvers apart from setting a time limit. Our goal does not include to compare both solvers. A x64-based computer with a processor Intel Core 2 Duo $(3.16 \mathrm{GHz})$ and 4 gigabytes of RAM was used in the experiments.

\subsection{Feasibility and Optimality}

All 112 WSRP instances are tackled using the model described in section 2 . We initially set the computational time to 15 minutes. For every instance, we record the time at which the first feasible solution is found, if such solution exits. Also, for instances with at least one feasible solution, we report on the gap reduction when the computation time limit is reached. If at any time the gap is 0, i.e. an optimal solution is found, that time is also recorded. Instances are grouped by the distribution of visits (clustered, random or mixed) and the size of the planning horizon. Knowing for which instances feasible solutions 


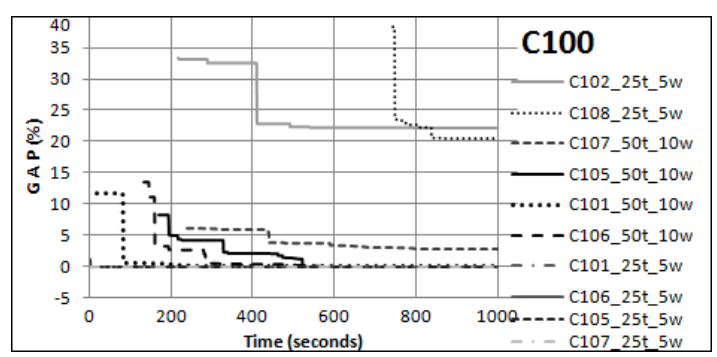

(a) $\mathrm{Cl} 00$ with all constraints and 15 min time limit.

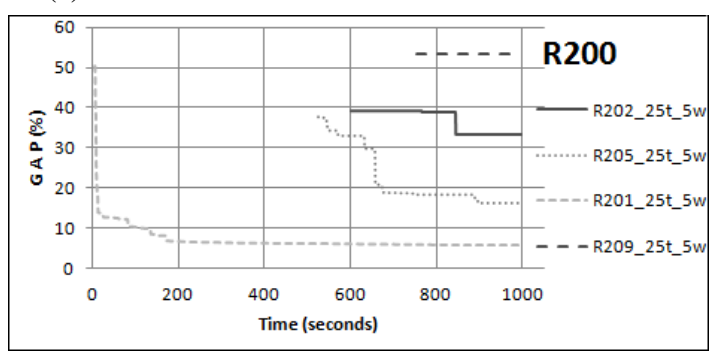

(c) R200 with all constraints and 15 min time limit.

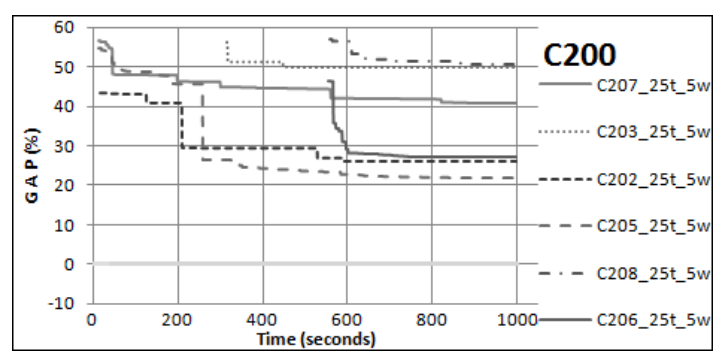

(b) C200 with all constraints and 15 min time limit.

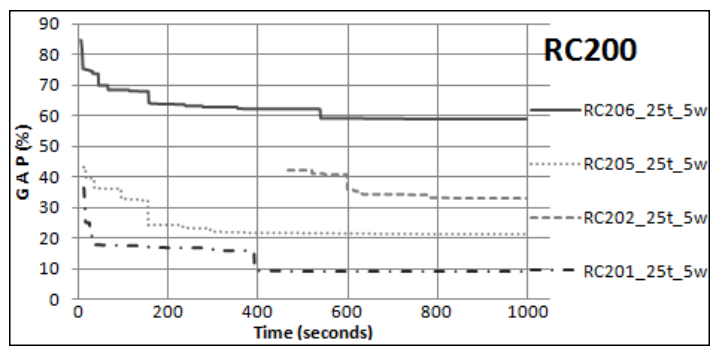

(d) RC200 with all constraints and 15 min time limit.

Figure 2: Results of experiments with time limit of 15 min showing gap reduction for feasible instances found (26 in total).

are achieved provides an insight into the clustering component of the visits since around a third of the instances have clustered visits e.g. C100 and C200.

In this first set of experiments, at least one feasible solution is achieved for 26 out of the 112 instances. From those 26 instances, the optimal solution (gap $0.0 \%$ ) is found for 7 of them. Figure 2 shows the gap reduction achieved within 15 minutes for the 26 instances. The figure shows one graph for each of the 4 groups (C100, C200, R200, RC200) with feasible solutions found. For the remaining 86 instances, infeasibility was reported by the solver for 7 instances. In the remaining 79 instances, Gurobi times out after 15 minutes with no additional information. No feasible solution is achieved within 15 minutes for any instance within the R100 and RC100 groups. Eventhoug all instances involve the same number of activities, groups R100 and RC100 seem harder to solve because they have a shorter planning horizon (230 and 240 minutes) when compared to the other four groups where the planning horizon is 900 minutes or more. The duration of the planning horizon also dictates employees' available working time, resulting in less capacity of working hours in such scenarios. Additionally, the random distribution of visits in these two (R) groups (see Figure 1) does not exploit the cluster feature that other scenarios present. Once an employee enters a cluster region it is very likely that he/she remains there for other visits rather than travelling to a different cluster location, saving time overall. Gurobi finds only 4 feasible solutions for instances in group R200 with randomly spread visits. All the 7 optimal
Table 1: Instances where Gurobi finds optimal solutions, showing two times: first feasible solution and optimal.

\begin{tabular}{|lll|lll|}
\hline Instance & Feasible & Optimal & Instance & Feasible & Optimal \\
\hline C101_25_5 & 0.62 & 0.62 & C105_25_5 & 2.0 & 3.86 \\
C105_50_10 & 168.0 & 522.08 & C106_25_5 & 0.67 & 0.67 \\
C107_25_5 & 2.0 & 2.78 & C201_25_5 & 1.0 & 1.63 \\
C201_50_10 & 43.0 & 44.99 & & & \\
\hline
\end{tabular}

Table 2: Infeasible instances and time required to prove infeasibility

\begin{tabular}{|l|l|l|l|}
\hline Instance & Time & Instance & Time \\
\hline R101_25_5 & 1.53 & R101_50_10 & 20.05 \\
R102_50_10 & 82.75 & R103_50_10 & 104.99 \\
R104_50_10 & 296.3 & R105_25_5 & 4.78 \\
RC102_50_10 & 188.63 & & \\
\hline
\end{tabular}

solutions that were found (see Table 1) are in the clustered groups (C) and the majority in group $\mathrm{C} 100$. This group has the second largest planning horizon of all instances (1236 minutes) giving more working time overall. All infeasible instances are shown in Table 2, almost all of them corresponding to group R100.

\subsection{Effect of Teaming and Connected Activities Constraints}

In these experiments we remove the constraints for teaming and connected activities from the $112 \mathrm{WSRP}$ instances. Therefore, all visits require one employee only and every activity is independent of the others. The other attributes, planning time horizon length, activities duration, activities time windows, etc. remain unchanged. We also allow 15 minutes of computational time for each instance. As in the previous experiments, we record the number of instances for which at least a feasible solution is achieved and the 
Table 3: Number of feasible solutions found in every group. Values in parentheses indicate the number of instances per group.

\begin{tabular}{|l|cccccccc|}
\hline Teaming and Connected Activities (TC) & Time Limit & C100(18) & C200(16) & R100(24) & R200(22) & RC100(16) & RC200(16) & Total(112) \\
\hline 1 with TC constraints & 15 min & 10 & 8 & 0 & 4 & 0 & 4 & 26 \\
2 without TC constraints & $15 \mathrm{~min}$ & 13 & 13 & 3 & 12 & 8 & 10 & 59 \\
\hline
\end{tabular}

corresponding rate of improvement (gap reduction). Our aim is to find out if more feasible solutions are found compared to the results from the previous experiments (subsection 4.2). If that is the case, it could suggest that teaming and connected activities make the problem harder to solve. Constraints for teaming and connected activities are considered together because a teaming constraint is modelled as a connected activity constraint of the synchronisation type. If a visit $x$ requires a team of $m$ workers, then $m-1$ virtual visits are created with the same requirements as the original visit $x$. Afterwards, a synchronisation constraint is created between each possible pair of visits. Such procedure guarantees that all employees required to perform visit $x$ arrive at the same time, effectively forming a team.

Table 3 shows the number of instances in each group for which at least one feasible solution was achieved within 15 minutes computation time. The first row summarises the results from the first set of experiments in the previous subsection. The second row summarises the results when teaming and connected activities constraints are relaxed. After comparing both rows it is clear that removing these constraints allows the solver to find feasible solutions for more instances. If we compare only the instances where a known feasible solution is known (from previous subsection 4.2), results in Table 4 show that significantly better gaps are achieved (approximately $10 \%$ overall) when the teaming and connected activities constraints are relaxed. Table 4 shows that the version of the problem instance without these constraints achieves a better gap than its complete counterpart. As it can be noticed, for the majority of instances the result is $=$ or $>$, with the exception of one instance (C106_50_10) but the difference is very small $(0.04 \%)$. Additionally, for 59 of the instances a feasible solution is achieved when both teaming and connected activities constraints are relaxed This is in contrast to the only 26 instances for which a feasible solution was found when both types of constrains are present.

In all groups of instances, removing the teaming and connected activities constraints produces an improvement in the number of instances achieving feasibility. Groups R100 and RC100 for which no feasible solution was found before, now have 3 and 8 instances with feasible solutions respectively. But overall, these groups still remain as the two groups with the least
Table 4: Feasible solutions with final gap achieved after 15 min computation time. The second (w/TC) and third (wo/TC) columns show gap results with and without the teaming and connected activities constraints respectively.

\begin{tabular}{|lll|lll|}
\hline Instance & w/TC & wo/TC & Instance & w/TC & wo/TC \\
\hline C101_25_5 & $0 \quad=$ & 0 & C101_50_10 & $0.07>0$ \\
C102_25_5 & $22.1>12.4$ & C105_25_5 & $0=0$ \\
C105_50_10 & $0.04>0$ & C106_25_5 & $0=0$ \\
C106_50_10 & $0.03<0.34$ & C107_25_5 & $0=0$ \\
C107_50_10 & $2.9>0$ & C108_25_5 & $20.4>4.55$ \\
C201_25_5 & $0=0$ & C201_50_10 & $0=0$ \\
C202_25_5 & $26.1>1.63$ & C203_25_5 & $49.7>25.4$ \\
C205_25_5 & $21.8>1.82$ & C206_25_5 & $27.2>1.56$ \\
C207_25_5 & $40.6>11.9$ & C208_25_5 & $50.5>16.5$ \\
R201_25_5 & $5.77>4.56$ & R202_25_5 & $33.3>16.3$ \\
R205_25_5 & $16.3>6.52$ & R209_25_5 & $53.2>14$ \\
RC201_25_5 & $9.29>0.84$ & RC202_25_5 & $32.9>31.5$ \\
RC205_25_5 & $21.2>19.6$ & RC206_25_5 & $58.8>44.9$ \\
\hline \multicolumn{5}{|c|}{ Mean w/TC: 18.93} & Mean wo/TC: 8.24 \\
\hline
\end{tabular}

number of feasible solutions reported. Such result confirm that even when the teaming and connected activities constraints are relaxed, a group of clustered visits is easier to solve than a random distribution of visiting locations. In general, our results for this second set of experiments suggest that the adaptations made to the Solomon instances to generate WSRP problem instances, make the problem harder for a current solver to tackle. Figure 3 shows the gap reduction for the instances achieving feasibility within $15 \mathrm{~min}$ utes (900 seconds) without teaming and connected activities constraints, grouped by location and planning horizon. In the next set of experiments, we extend the computation time given to the solver in order to investigate the rate of improvement in the gaps and find more feasible solutions.

\subsection{Extending Computational Time}

In this set of experiments we increase the computation time from 15 to 60 minutes and then up to 240 minutes. Our objective is to further investigate feasibility and optimality in the WSRP instances with extended computation time available for the mathematical programming solver. In the results of the first set of experiments (section 4.2) Gurobi timed out in 79 instances without finding feasible solutions for any of them. In this third set of experiments, those 79 instances, without conclusive results before, are tackled once more using 60 minutes and 240 minutes of computation time. A computation time beyond 240 minutes is not practical for a problem with a planning horizon of 1 day in the majority of WSRP scenarios. 


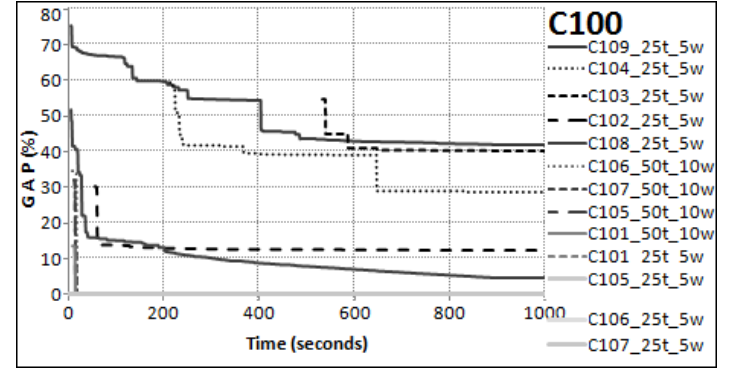

(a) $\mathrm{Cl} 00$ without $\mathrm{TC}$ constraints.

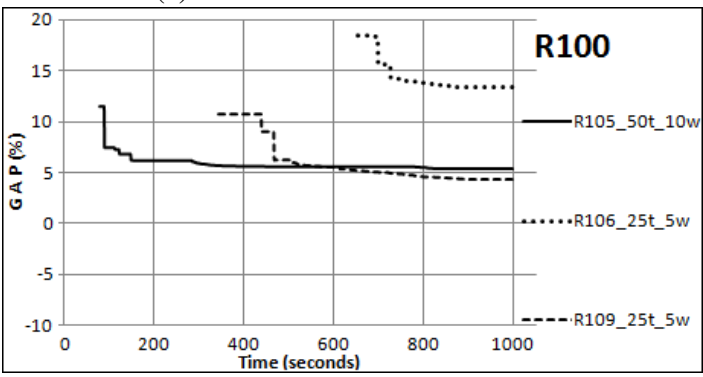

(c) R100 without TC constraints.

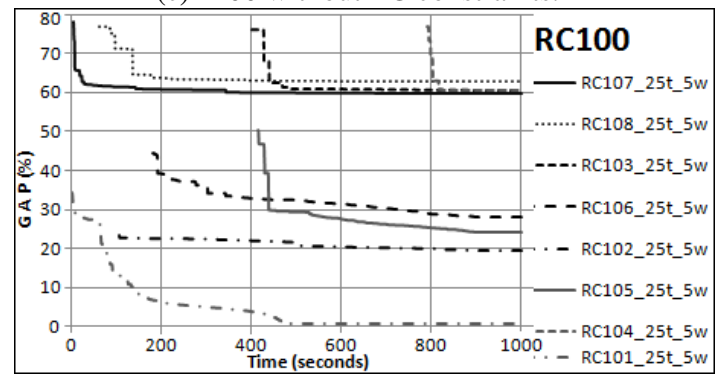

(e) RC100 without TC constraints.

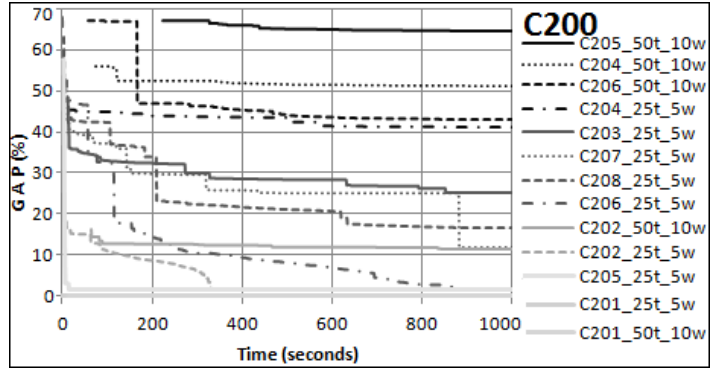

(b) C200 without TC constraints.

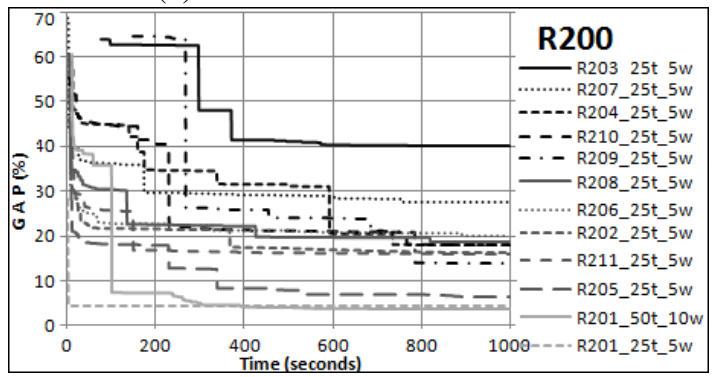

(d) R200 without TC constraints.

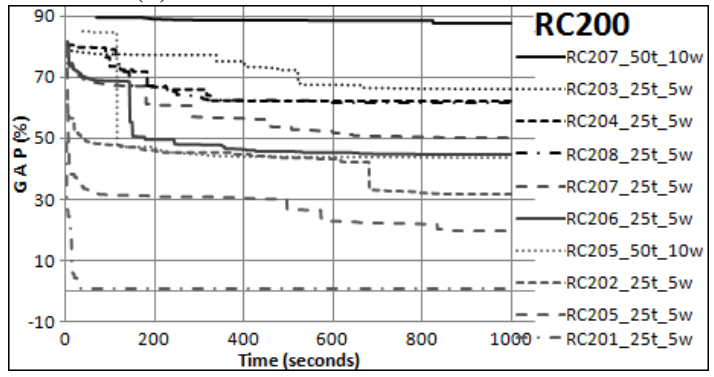

(f) RC200 without TC constraints.

Figure 3: Gap reduction of feasible solutions for instances without Teaming and Connected activities (TC) constraints.

Table 5: Instances for which feasible solutions were found within the time limit of 60 minutes. The time in seconds at which Gurobi found the first feasible solution is shown, together with the initial gap and final gap values.

\begin{tabular}{|c|c|c|c|}
\hline Instance & 1st feasible & Initial & Final \\
\hline R201_50_10 & 2594 & $18.6 \%$ & $12.3 \%$ \\
R210_25_5 & 2099 & $42.6 \%$ & $31.7 \%$ \\
RC106_25_5 & 1915 & $40.9 \%$ & $39.1 \%$ \\
\hline
\end{tabular}

Increasing the computational time to 60 minutes for the 79 instances produced feasible solutions for 3 of those instances, none of them achieving optimality. The remaining 76 instances again timed out with no further information. Details of these additional results is shown in Table 5. It should be noted that the computation time for finding the first feasible solution for the 3 instances is more than 30 minutes (1800 seconds). The difference between the initial gap and the final gap values is less than $11 \%$. Figure 4 shows the gap reduction achieved over the given computation time.

We then increased the computation time from 60

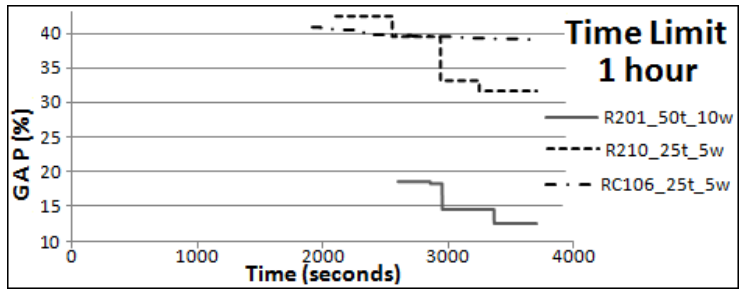

Figure 4: Gap reduction of instances for which feasible solutions are achieved within the time limit of 60 minutes.

to 240 minutes, aiming to find out the practical limits (assuming that more than 4 hours of computation time is not practical) of a current solver when tackling difficult WSRP instances. Feasible solutions were found for 16 instances. Table 6 provides information about these results. Rows marked with an (*) indicate instances for which feasible solutions were found before 3600 seconds (60 $\mathrm{min}$ ), but for which no feasible solution had been reported in the previous experiments (with 15 and 60 minutes). This could be explained by the search strategy used by Gurobi, as the frequency of using heuristics and how deep it goes 
Table 6: Feasible solutions and initial/final gap achieved after 240 min computation time (first solution time shown in seconds). For all these instances no feasible solution was found in the previous experiments with 15 and 60 minutes.

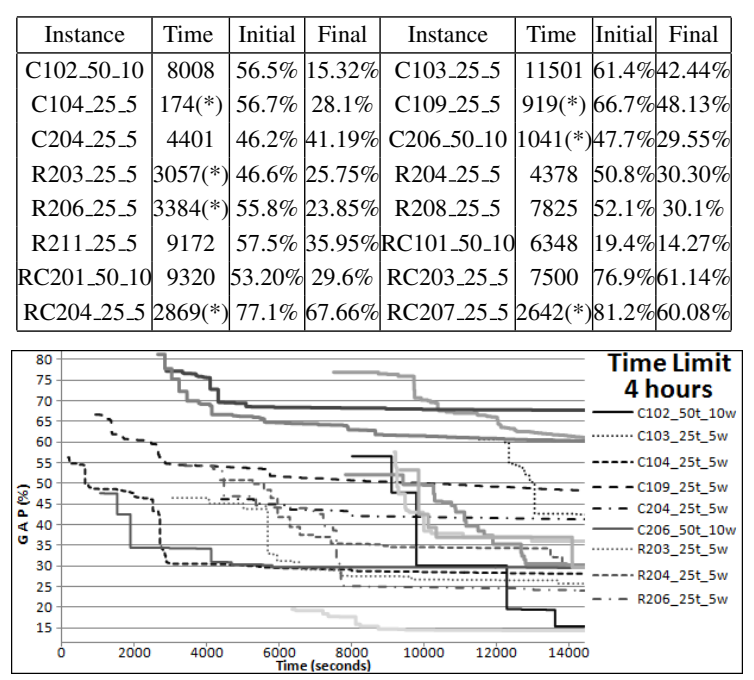

Figure 5: Gap reduction of instances for which feasible solutions are achieved within the time limit of 240 minutes.

into the branching tree might depend on the computation time available. Figure 5 complements Table 6 as a visual representation of the gap reduction over the 240 minutes for the instances that achieve feasible solutions. A reduction of $15 \%$ in gap when compared to the initial solution is achieved for all instances except RC101_50_10. As noted before, for some instances a first feasible solution is found before 60 minutes, rows marked with $(*)$ in Table 6 , which shows that analysing the first hour of computation results in a four-hour experiment is not equivalent to restricting the computation time to only one hour with Gurobi.

\subsection{Methods Used by Gurobi}

Gurobi provides information regarding the current gap achieved while performing the optimisation. In our experiments, we set up the solver to report the gap reduction every 15 seconds. When a gap reduction is achieved, the method used is reported by the solver. The objective in this set of experiments is to identify which method is used by Gurobi when finding better solutions for each instance. For every new feasible solution Gurobi reports whether the solution was found by branching or by heuristics (Inc., 2013). If most of the time new feasible solutions are found this is achieved by heuristics, it would justify developing our own. In all previous experiments, without exemption, Gurobi found more gap improvements when using heuristics. It is expected that MIP heuristics find more feasible solutions than the branching process for the VRPTW. The adaptations to the data set and modification of the VRPTW model to tackle WSRP have
Table 7: Summary of methods used by Gurobi during the optimisation process. Columns $\mathrm{H} / \mathrm{B}$ report the number of gap reductions within a group of instances that are achieved with Heuristics $(\mathrm{H})$ or Branching $(\mathrm{B})$. Within every group the number of instances with feasible solutions is reported.

\begin{tabular}{|lllllll|}
\hline Time(\#) & C100 & H/B & C200 & H/B & R100 & H/B \\
\hline $15 \mathrm{~m}(112)$ & 10 & $26 / 16$ & 8 & $27 / 11$ & 0 & $0 / 0$ \\
$* 15 \mathrm{~m}(112)$ & 13 & $50 / 12$ & 13 & $71 / 19$ & 3 & $10 / 5$ \\
$60 \mathrm{~m}(79)$ & 0 & $-/-$ & 0 & $-/-$ & 0 & $-/-$ \\
$240 \mathrm{~m}(112)$ & 14 & $95 / 23$ & 10 & $83 / 23$ & 0 & $0 / 0$ \\
\hline $\operatorname{Time}(\#)$ & $\mathrm{R} 200$ & $\mathrm{H} / \mathrm{B}$ & $\mathrm{RC} 100$ & $\mathrm{H} / \mathrm{B}$ & $\mathrm{RC} 200$ & $\mathrm{H} / \mathrm{B}$ \\
\hline $15 \mathrm{~m}(112)$ & 4 & $25 / 10$ & 0 & $0 / 0$ & 4 & $57 / 14$ \\
$* 15 \mathrm{~m}(112)$ & 12 & $75 / 21$ & 8 & $48 / 25$ & 10 & $99 / 20$ \\
$60 \mathrm{~m}(79)$ & 2 & $8 / 1$ & 1 & $3 / 2$ & 0 & $-/-$ \\
$240 \mathrm{~m}(112)$ & 11 & $196 / 27$ & 2 & $11 / 1$ & 8 & $126 / 15$ \\
\hline
\end{tabular}

similar results. In fact, the number of times a heuristic within Gurobi finds a better solution is in general larger for instances that include the additional constraints in the WSRP instances.

Table 7 summarises the number of times a gap reduction was achieved for every group of instances in all experiments. The table has four rows but split in two parts vertically, each part has three groups of instances. Note that the second row in each part, marked with $\left(^{*}\right)$, refers to all instances without the teaming and connected activities constraints. The third row in each part shows the 79 instances that timed out after 15 minutes in the first set of experiments but then executed for up to 60 minutes. The number in parentheses after the time limit is the number of instances used in that set of experiments. In all groups there are more gap reductions achieved by heuristics than by branching (H/B values).

\section{CONCLUSION}

In this study we applied a vehicle routing problem with time windows (VRPTW) model that incorporates temporal precedence and synchronisation constraints to tackle the workforce scheduling and routing problem (WSRP). We extended 112 well-known VRPTW instances to generate a set of 112 difficult WSRP instances. Using a current powerful optimisation solver, only some of the generated WSRP instances were tackled with some success. For 60 instances, the solver could not even find a feasible solution (see Table 8). This suggests that alternative solution methods should be considered besides existing mathematical programming solvers.

This computational study provides solid evidence than the WSRP problem instances are more challenging than the VRPTW ones from which they were generated. Our experiments provide an insight into what makes this problem more difficult and also provides an updated perspective on the practical applicability of existing optimisation solvers to tackle scenarios involving the scheduling and routing of workforce. The 
Table 8: Results summary for all experiments. Gurobi did not find a feasible solution for 60 instances.

\begin{tabular}{|c|c|c|c|c|c|c|c|}
\hline Set & Feasible Infeasible & $\begin{array}{c}\text { Not } \\
\text { found }\end{array}$ & Set & Feasible Infeasible & $\begin{array}{c}\text { Not } \\
\text { found }\end{array}$ \\
\hline C100 & 14 & 0 & 4 & C200 & 10 & 0 & 6 \\
R100 & 0 & 6 & 18 & R200 & 11 & 0 & 11 \\
RC100 & 2 & 1 & 13 & RC200 & 8 & 0 & 8 \\
\hline Totals & \multicolumn{7}{|c|}{ Feasible 45 Infeasible 7 } \\
\hline
\end{tabular}

generated WSRP instances are more difficult to solve due to the additional teaming and connected activities constraints (similar results are reported by Rasmussen et al. (2012) ). Additionally, we found that WSRP instances with clustered visiting locations tend to be easier to solve according to the gap reduction rate reported by the solver in our experiments.

Finally, we established that the computational time for a mathematical solver to find good feasible solutions for the generated WSRP problem instances needs to be more than 1 hour. Considering only the 45 instances for which feasible solutions were found, for 29 of them feasible solutions were found within an hour. For the reminder 16, feasible solutions were found within 1 to 4 hours. Nevertheless, for $90 \%$ of the instances, feasible solutions were found within 2 hours and 5 minutes. Adding two more hours of computational time achieved only $10 \%$ more feasible solutions. We consider this not to be practical, hence suggest a maximum computation time of 2 hours when solving WSRP instances with planning time horizon of one day.

\section{REFERENCES}

Akjiratikarl, C., Yenradee, P., and Drake, P. R. (2007). Pso-based algorithm for home care worker scheduling in the uk. Computers \& Industrial Engineering, 53(4):559-583.

An, Y.-J., Kim, Y.-D., Jeong, B., and Kim, S.-D. (2012). Scheduling healthcare services in a home healthcare system. Journal of the Operational Research Society, 63:1589-1599.

Bertels, S. and Fahle, T. (2006). A hybrid setup for a hybrid scenario: combining heuristics for the home health care problem. Computers \& Operations Research, 33(10):2866-2890.

Bredström, D. and Rönnqvist, M. (2008). Combined vehicle routing and scheduling with temporal precedence and synchronization constraints. European Journal of Operational Research, 191(1):19-31.

Castillo-Salazar, J. A., Landa-Silva, D., and Qu, R. (2012). A survey on workforce scheduling and routing problems. In Proceedings of the 9th International Conference on the Practice and Theory of Automated Timetabling, pages 283-302.

Cheng, E. and Rich, J. L. (1998). A home health care routing and scheduling problem. Technical Report TR9804, Rice University, Texas.

Chuin Lau, H. and Gunawan, A. (2012). The patrol schedul- ing problem. In Proceedings of the 9th International Conference on the Practice and Theory of Automated Timetabling, pages 175-192.

Chuin Lau, H., Sim, M., and Meng Teo, K. (2003). Vehicle routing problem with time windows and a limited number of vehicles. European Journal of Operational Research, 148:559-569.

Dohn, A., Kolind, E., and Clausen, J. (2009). The manpower allocation problem with time windows and jobteaming constraints: A branch-and-price approach. Computers \& Operations Research, 36(4):11451157.

Dondo, R. and Cerdá, J. (2007). A cluster-based optimization approach for the multi-depot heterogeneous fleet vehicle routing problem with time windows. $E u$ ropean Journal of Operational Research, 176:14781507.

Glover, F. and McMillan, C. (1986). The general employee scheduling problem. an integration of $\mathrm{ms}$ and ai. Computers \& Operations Research, 13(5):563-573.

Günther, M. and Nissen, V. (2012). Application of particle swarm optimization to the british telecom workforce scheduling problem. In Proceedings of the 9th International Conference on the Practice and Theory of Automated Timetabling, pages 242-256.

Inc., G. O. (2013). Gurobi Optimizer, Reference Manual version 5.5. Gurobi Optimization Inc.

Korsah, G. A., Stentz, A., Dias, M. B., and Aslam, I. (2010). Optimal vehicle routing and scheduling with precedence constraints and location choice. In IEEE International Conference on Robotics and Automation Workshop on Intelligent Autonomous Systems.

Li, Y., Lim, A., and Rodrigues, B. (2005). Manpower allocation with time windows and job-teaming constraints. Naval Research Logistics, 52(4):302-311.

Misir, M., Smet, P., Verbeeck, K., and Vanden Bergue, G. (2011). Security personnel routing and rostering: a hyper-heuristic approach. In Proceedings of the $3 \mathrm{rd}$ International Conference on Applied Operational Research, pages 193-206.

Nickel, S., Schröder, M., and Steeg, J. (2012). Mid-term abd short-term planning support for home health care services. European Journal of Operational Research, 219:574-587.

Rasmussen, M. S., Justesen, T., Dohn, A., and Larsen, J. (2012). The home care crew scheduling problem: Preference-based visit clustering and temporal dependencies. European Journal of Operational Research, 219(3):598-610.

Solomon, M. M. (1987). Algorithms for the vehicle routing and scheduling problems with time windows constraints. Operations Research, 35(2):254-265.

Taillard, E. D., Laporte, G., and Gendreau, M. (1996). Vehicle routeing with multiple use of vehicles. Journal of the Operational Research Society, 47:10651070.

Toth, P. and Vigo, D. (1987). The vehicle routing problem, volume 9. Society for Industrial and Applied Mathematics. 